\title{
Pflegestärkungsgesetz
}

\section{Bundestag verabschiedet Pflegegesetz II}

- Der Deutsche Bundestag hat am 13. November 2015 das Zweite Pflegestärkungsgesetz (PSG II) beschlossen. Das Gesetz tritt am 1. Januar 2016 in Kraft. Der Deutsche Pflegerat (DPR) spricht von einer Vielzahl an guten und wichtigen Neuregelungen, äußert aber auch Kritik. „Mit dem neuen Pflegebedürftigkeitsbegriff gehören Minutenpflege und Defizitorientierung bald der Vergangenheit an", sagt der Pflegebevollmächtigte der Bundesregierung, Staatssekretär Karl-Josef Laumann. „Stattdessen wird es eine Begutachtung geben, die ganz

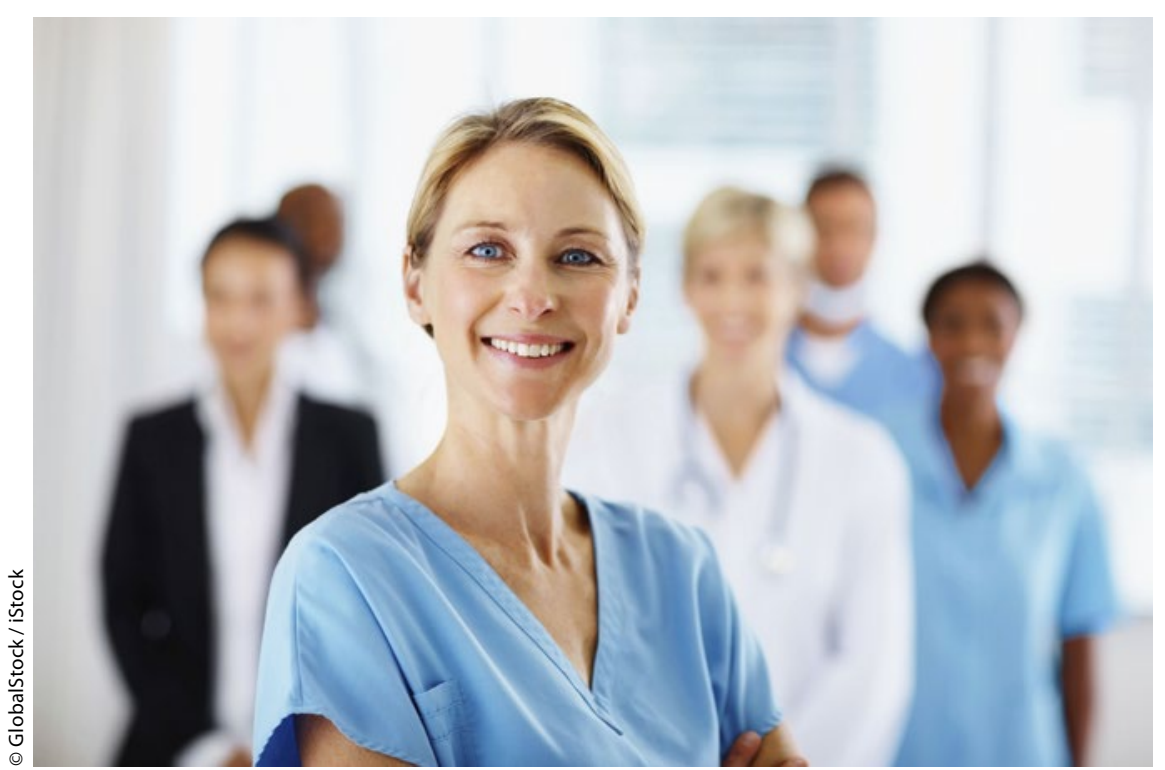

individuell beim einzelnen Menschen schaut, wie selbstständig er seinen Alltag noch gestalten kann. " Das sei ein Quantensprung. Zudem bekomme der Pflege-TÜV in seiner jetzigen Form ein klares Verfallsdatum. Es werde spätestens ab 2018 ein neues Qualitätsprüfungs- und Transparenzsystem geben, das den Bürgern endlich eine echte Orientierungshilfe biete.

Der neue Pflegebegriff und das neue Begutachtungsverfahren werden zum 1. Januar 2017 wirksam. Wichtige Verbesserungen für Pflegebedürftige und ihre Angehö- rigen treten bereits zum 1.Januar 2016 in Kraft.

Der Pflegerat begrüßt das Pflegestärkungsgesetz II, vor allem die Einführung eines neuen Pflegebedürftigkeitsbegriffes. Hierzu erklärt DPR-Präsident Andreas Westerfellhaus: „Die damit erhoffte Verbesserung der pflegerischen Versorgung wird jedoch ausbleiben, wenn es nicht umgehend zu konkreten Regelungen für mehr Personal kommt. An dieser Stelle hat der Gesetzgeber gepasst." Er habe die Beantwortung der Frage, mit welchem Personal die erhofften Mehrleistungen erbracht werden sollen, außen vor gelassen. Das sei Westerfellhaus zufolge die größte Schwachstelle des Gesetzes. Der Pflegerat fordere bundeseinheitliche Personalschlüssel in stationären Pflegeeinrichtungen. „Es ist nicht nachvollziehbar, dass die heutige Personalausstattung angesichts bundeseinheitlich geregelter Module für die Pflegebedürftigkeit - wie auch für die Leistungen - je nach Bundesland um mehr als 20\% in der Pflege abweicht", so der DPR-Präsident. Das habe direkte Auswirkungen auf die Qualität der Versorgung und die Arbeitsbelastung der Mitarbeiter. "Die Chance, endlich einen Schlussstrich unter diesen Flickenteppich zu ziehen, wurde jetzt verpasst."

www.bmg.bund.de

\section{Modellprojekt \\ Gemeindeschwester ${ }^{\text {plus }}$}

— In Koblenz hat am 9. November die erste Pflegefachkraft im Projekt Gemeindeschwesterplus ihre Arbeit aufgenommen. In dieser Funktion kümmert sie sich um hochbetagte Menschen, die nicht pflegebedürftig sind, und besucht und berät sie auf Wunsch in ihrem häuslichen Umfeld.

Das Modellprojekt der rheinland-pfälzischen Landesregierung wird zurzeit in neun Landkreisen und kreisfreien Städten erprobt.

www.msagd.rlp.de

\section{Onkologie}

\section{Patientenorientierung im Fokus}

— Eines der zentralen Themen der Mitgliederversammlung des Berufsverband der Niedergelassenen Hämatologen und Onkologen in Deutschland BNHO e.V. war die Qualität der Versorgung onkologischer Patienten. Zu den Schwerpunkten der Verbandsarbeit wird die Umsetzung einer noch stärkeren Patientenorientierung gehören. Hierzu wurde bereits Anfang des Jahres ein 5-Punkte-Plan vorgelegt. Er zielt darauf ab, einer Zersplitterung der Verantwortung im Bereich Versorgung entgegenzuwirken und die Kontinuität auch in der Nachsorge zu gewährleisten. Weiter wird es darum gehen, die psychoonkologische Grundversorgung zu optimieren sowie den Zugang zu innovativen Therapieoptionen weiterhin zu sichern.

Hintergrund ist die steigende Komplexität des Krankheitsbildes in der Onkologie sowie die zunehmende Zahl der von niedergelassenen Krebsspezialisten zu versorgenden Patienten. Die im BNHO zusammengeschlossenen Hämatologen und Onkologen behandeln jährlich gut 600.000 an Krebs erkrankte Patienten.

www.bnho.de 